\title{
The equivariant topology of stable Kneser graphs
}

\author{
Carsten Schultz
}

Institut für Mathematik, Freie Universität Berlin, Berlin, Germany

\begin{abstract}
Schrijver introduced the stable Kneser graph $S G_{n, k}, n \geq 1, k \geq 0$. This graph is a vertex critical graph with chromatic number $k+2$, its vertices are certain subsets of a set of cardinality $m=2 n+k$. Björner and de Longueville have shown that its box complex is homotopy equivalent to a sphere, $\operatorname{Hom}\left(K_{2}, S G_{n, k}\right) \simeq \mathbb{S}^{k}$. The dihedral group $D_{2 m}$ acts canonically on $S G_{n, k}$. We study the $D_{2 m}$ action on $\operatorname{Hom}\left(K_{2}, S G_{n, k}\right)$ and define a corresponding orthogonal action on $\mathbb{R}^{k+1} \supset \mathbb{S}^{k}$. We establish a close equivariant relationship between the graphs $S G_{n, k}$ and Borsuk graphs of the $k$-sphere and use this together with calculations in the $\mathbb{Z}_{2}$-cohomology ring of $D_{2 m}$ to tell which stable Kneser graphs are test graphs in the sense of Babson and Kozlov.

The graphs $S G_{2 s, 4}$ are test graphs, i.e. for every graph $H$ and $r \geq 0$ such that $\operatorname{Hom}\left(S G_{2 s, 4}, H\right)$ is $(r-1)$-connected, the chromatic number $\chi(H)$ is at least $r+6$. On the other hand, if $k \notin\{0,1,2,4,8\}$ and $n \geq N(k)$ then $S G_{n, k}$ is not a homotopy test graph, i.e. there are a graph $G$ and an $r \geq 1$ such that $\operatorname{Hom}\left(S G_{n, k}, G\right)$ is $(r-1)$-connected and $\chi(G)<r+k+2$. The latter result also depends on a new necessary criterion for being a test graph, which involves the automorphism group of the graph.

Résumé. Schrijver a défini le graphe de Kneser stable $S G_{n, k}$, avec $n \geq 1$ et $k \geq 0$. Le graphe $S G_{n, k}$ est un graphe critique (par rapport aux sommets) de nombre chromatique $k+2$, dont les sommets correspondent à certains sousensembles d'un ensemble de cardinalité $m=2 n+k$. Björner et de Longueville ont démontré que son complexe de boîtes et la sphère sont homotopiquement équivalents, c'est-à-dire $\operatorname{Hom}\left(K_{2}, S G_{n, k}\right) \simeq \mathbb{S}^{k}$. Le groupe diédral $D_{2 m}$ agit sur $S G_{n, k}$ canoniquement. Nous étudions l'action de $D_{2 m} \operatorname{sur} \operatorname{Hom}\left(K_{2}, S G_{n, k}\right)$ et nous définissons une action orthogonale correspondante sur $\mathbb{R}^{k+1} \supset \mathbb{S}^{k}$. Par ailleurs, nous fournissons une relation équivariante étroite entre les graphes $S G_{n, k}$ et les graphes de Borsuk de la sphère de dimension $k$. Utilisant cette relation et certains calculs dans l'anneau de cohomologie de $D_{2 m}$ sur $\mathbb{Z}_{2}$, nous décrivons quels graphes de Kneser stables sont des graphes de tests selon la notion de Babson et Kozlov.

Les graphes $S G_{2 s, 4}$ sont des graphes de tests, c'est-à-dire que pour tout $H$ et $r \geq 0$ tels que $\operatorname{Hom}\left(S G_{2 s, 4}, H\right)$ est $(r-1)$-connexe, le nombre chromatique $\chi(H)$ est au moins $r+6$. D'autre part, si $k \notin\{0,1,2,4,8\}$ et $n \geq N(k)$, alors $S G_{n, k}$ n'est pas un graphe de tests d'homologie: il existe un graphe $G$ et un entier $r \geq 1$ tels que $\operatorname{Hom}\left(S G_{n, k}, G\right)$ est $(r-1)$-connexe et $\chi(G)<r+k+2$. Ce dernier résultat dépend d'un nouveau critère nécessaire pour être un graphe de tests, qui implique le groupe d'automorphismes du graphe.
\end{abstract}

Keywords: stable Kneser graph, Hom complex, graph homomorphism, test graph, alternating oriented matroid 


\section{Introduction}

\section{Background}

The subject of topological obstructions to graph colourings was started when Lovász determined the chromatic numbers of Kneser graphs in Lovász (1978).

1.1 Definition. Let $n \geq 1, k \geq 0$. The Kneser graph $K G_{n, k}$ is a graph with vertices the $n$-element subsets of a fixed set of cardinality $2 n+k$, say

$$
V\left(K G_{n, k}\right)=\left\{S \subset \mathbb{Z}_{2 n+k}:|S|=n\right\} .
$$

Two such sets are neighbours in $K G_{n, k}$ if and only if they are disjoint,

$$
E\left(K G_{n, k}\right)=\left\{(S, T) \in V\left(K G_{n, k}\right): S \cap T=\emptyset\right\} .
$$

It is easy to see that $K G_{n, k}$ admits a $(k+2)$-colouring, $\chi\left(K G_{n, k}\right) \leq k+2$. Lovász assigned to each graph $G$ a simplicial complex, its neighbourhood complex $\mathcal{N}(G)$ and proved the following two theorems.

1.2 Theorem. If $G$ is a graph and $r \geq 0$ such that $\mathcal{N}(G)$ is $(r-1)$-connected, then $\chi(G) \geq r+2$.

1.3 Theorem. The complex $\mathcal{N}\left(K G_{n, k}\right)$ is $(k-1)$-connected.

These establish $\chi\left(K G_{n, k}\right)=k+2$ as conjectured by Kneser.

The proof of Theorem 1.2 uses the Borsuk-Ulam theorem. This led Bárány to a simpler proof of $\chi\left(K G_{n, k}\right) \geq k+2$, which does not use any graph complexes but applies the Borsuk-Ulam theorem more directly Bárány (1978). This proof uses the existence of certain generic configurations of vectors in $\mathbb{R}^{k+1}$. Using a specific configuration of this kind, Schrijver found an induced subgraph of $K G_{n, k}$, the graph $S G_{n, k}$, with the property that already $\chi\left(S G_{n, k}\right)=k+2 \operatorname{Schrijver}(1978)$.

1.4 Definition. The stable Kneser graph $S G_{n, k}$ is the induced subgraph of $K G_{n, k}$ on the vertex set

$$
V\left(S G_{n, k}\right)=\left\{S \in V\left(K G_{n, k}\right):\{i, i+1\} \not \subset S \text { for all } i \in \mathbb{Z}_{2 n+k}\right\} .
$$

The vertices of $S G_{n, k}$ are called stable subsets of $\mathbb{Z}_{2 n+k}$.

Schrijver also proves that the graph $S G_{n, k}$ is vertex critical in the sense that it becomes $(k+1)$ colourable if an arbitrary vertex is removed.

A more systematic treatment of topological obstructions to the existence of graph colourings was suggested by Lovász and started by Babson and Kozlov (2006). For graphs $G$ and $H$, they define a cell complex $\operatorname{Hom}(H, G)$. The vertices of $\operatorname{Hom}(H, G)$ are the graph homomorphisms from $H$ to $G$. They introduce the concept of a test graph.

1.5 Definition. A graph $T$ is a homotopy test graph if for all loopless graphs $G$ and $r \geq 0$ such that $\operatorname{Hom}(T, G)$ is $(r-1)$-connected the inequality $\chi(G) \geq \chi(T)+r$ holds.

Since the complex $\operatorname{Hom}\left(K_{2}, G\right)$ is homotopy equivalent to $\mathcal{N}(G)$, Theorem 1.2 states that $K_{2}$ is a homotopy test graph. In Babson and Kozlov (2006) this result is extended to all complete graphs and in Babson and Kozlov (2007) to odd cycles. These proofs show a graph $T$ to be a test graph by studying the spaces $\operatorname{Hom}\left(T, K_{n}\right)$ and $C_{2}$-actions on them induced by a $C_{2}$-action on $T$. Here $C_{2}$ denotes the cyclic group of order 2 . Indeed, for a graph $T$ with an action of a group $\Gamma$ one can define the property of being a 
Г-test graph (Definition 3.1), which implies being a homotopy test graphs, and the homotopy test graphs mentioned above are shown to be $C_{2}$-test graphs.

In Schultz (2009) easier proofs, in particular for odd cycles, are obtained by instead studying the complex $\operatorname{Hom}\left(K_{2}, T\right)$ together with two involutions, one induced by the non-trivial involution of $K_{2}$ and another by an involution on $T$. This also yielded the somewhat isolated result that $K G_{2 s, 2}$ is a $C_{2}$-test graph. All known test graphs at that point were Kneser graphs or stable Kneser graphs, since $K G_{1, k}=S G_{1, k}$ is a complete graph on $k+2$ vertices and $S G_{n, 1}$ is a cycle of length $2 n+1$

In Dochtermann and Schultz (2010) it was shown that test graphs $T$ can be obtained by constructing graphs $T$ with prescribed topology of $\operatorname{Hom}\left(K_{2}, T\right)$.

\section{Overview and results}

Since all "naturally occurring" graphs which have so far been identified as test graphs are stable Kneser graphs (the result for $K G_{2 s, 4}$ can be derived from one for $S G_{2 s, 4}$ ), it is natural to ask if more or even all stable Kneser graphs are test graphs. Our main goal in this work is to decide which of them are test graphs. We can answer this question to a large extent. We find new test graphs, but it turns out that most stable Kneser graphs are not test graphs. We point out some of the results that we obtain on the way.

It has been known that stable Kneser graphs are related to spheres, for example Björner and de Longueville (2003) have shown the homotopy equivalence $\mathcal{N}\left(S G_{n, k}\right) \simeq \mathbb{S}^{k}$. We describe more aspects of this relationship and also make it equivariant. Braun (2010) has shown that for $n>1$ the automorphism group of $S G_{n, k}$ is the dihedral group $D_{2 m}$ with $2 m$ elements, $m=2 n+k$. In Section 2 we define a $(k+1)$-dimensional orthogonal representation of $D_{2 m}$ and an explicit map from its unit sphere to $\operatorname{Hom}\left(K_{2}, S G_{n, k}\right)$,

$$
\mathbb{S}\left(W_{n, k}\right) \rightarrow_{C_{2} \times D_{2 m}} \operatorname{Hom}\left(K_{2}, S G_{n, k}\right) .
$$

This map is equivariant with respect to the action of the dihedral group, and also with respect to the 2-element group $C_{2}$ acting as the antipodal map on the sphere and via $K_{2}$ on $\operatorname{Hom}\left(K_{2}, S G_{n, k}\right)$. This map is a $D_{2 m}$-homotopy equivalence. Its construction involves the alternating oriented matroid. It uses a connection between the approaches of Bárány (1978) and Schrijver (1978) on one hand and Lovász (1978) on the other, which has not been made explicit previously.

A construction which is in some sense dual to (1) is best formulated using the notion of a Borsuk graph. We write $C_{2}=\{e, \tau\}$.

1.6 Definition. Let $(X, d)$ be a metric space with an isometric $C_{2}$-action and $\varepsilon>0$. We define the $\varepsilon$-Borsuk graph of $X, B_{\varepsilon}(X)$, as follows. The vertex set of $B_{\varepsilon}(X)$ is the set of all points of $X$ and $x \sim y \Longleftrightarrow d(x, \tau y)<\varepsilon$.

1.7 Theorem (Proposition 5.3). Let $k \geq 0$ and $\varepsilon>0$. Then for large enough $n$ and $m=2 n+k$ there is an equivariant graph homomorphism

$$
S G_{n, k} \rightarrow_{D_{2 m}} B_{\varepsilon}\left(\mathbb{S}\left(W_{n, k}\right)\right)
$$

The first construction will help us to show that certain stable Kneser graphs are test graphs, the second that certain stable Kneser graphs are not. These constructions are also dual in the way that the first uses properties of covectors of the alternating oriented matroid and the second uses properties of its vectors.

The $D_{2 m}$-representation $W_{n, k}$ gives rise to a $(k+1)$-dimensional vector bundle $\xi_{n, k}$ over the classifying space $B D_{2 m}$, Definition 4.2 This in turn defines Stiefel-Whitney classes $w_{i}\left(\xi_{n, k}\right) \in H^{i}\left(D_{2 m} ; \mathbb{Z}_{2}\right)$ and 
also classes $\bar{w}_{i}\left(\xi_{n, k}\right) \in H^{i}\left(D_{2 m} ; \mathbb{Z}_{2}\right)$ by $\sum_{i \geq 0} w_{i} \cdot \sum_{i \geq 0} \bar{w}_{i}=1$. An approach relying on the map (1) and similar to that in Schultz (2009), but with different topological tools, yields:

1.8 Proposition Section 4). Let $n, k \geq 1, m=2 n+k$. If $\bar{w}_{r}\left(\xi_{n, k}\right) \neq 0$ for all $r \geq 1$, then $S G_{n, k}$ is a $D_{2 m}$-test graph and hence a homotopy test graph.

Calculations in the cohomology ring $H^{*}\left(D_{2 m} ; \mathbb{Z}_{2}\right)$ identify the cases in which this criterion is applicable.

1.9 Theorem (see Corollary 4.4). Let $n, k \geq 0$. If $k \in\{0,1,2\}$ or if $k=4$ and $n$ is even, then $S G_{n, k}$ is a homotopy test graph.

Here, mainly the case $k=4, n$ even, is new, and for it, it would not have been sufficent to restrict the calculations to a 2-element subgroup of $D_{2 m}$. Consequently, this result could not have been obtained by a direct application of the test graph criteria given in Schultz (2009).

So far, all proofs that certain graphs are test graphs used actions of a group on this graph. We show that this is not merely because it was the only known technique.

1.10 Theorem (see Theorem 3.3). Let $T$ be a finite, connected, vertex critical graph. Then $T$ is a homotopy test graph if and only if $T$ is an Aut $(T)$-test graph.

This also gives us a tool to prove that graphs are not test graphs. For example we note the curious consequence that a connected vertex critical graph without non-trivial automorphisms cannot be a test graph.

Using Theorem 1.7 some algebraic topology of vector bundles and a few constructions in the category of graphs, we obtain the following criterion.

1.11 Proposition (Proposition 6.3). Let $k \geq 1$. Then there is an $N>0$ such that for all $n \geq N$ the following holds: If there is an $r>0$ such that $\bar{w}_{r}\left(\xi_{n, k}\right)=0$ and $r=1$ or $r \equiv 0(\bmod 2)$, then $S G_{n, k}$ is not a $D_{2 m}$-test graph and hence not a homotopy test graph.

Again we do some calculations and arrive at the following result.

1.12 Theorem. Let $k \geq 0, k \notin\{0,1,2,4,8\}$. Then there is an $N>0$ such that for $n o n \geq N$ the graph $S G_{n, k}$ is a homotopy test graph.

Also, there is an $N>0$ such that for no odd $n \geq N$ the graph $S G_{n, 8}$ is a homotopy test graph.

This text is an extended abstract of Schultz (2010), and we will concentrate here on the constructions that we deem interesting and omit almost all calculations.

\section{Definitions and notation}

We will use the same terminology and notation as in Dochtermann and Schultz (2010) and refer the reader there for details.

Our graphs are undirected and without multiple edges. Even though we are mostly interested in graphs without loops, allowing loops is important for some constructions. We call a graph without loops loopless, and one in which every vertex is looped we call reflexive. A graph homomorphism $f: G \rightarrow H$ is a function $f: V(G) \rightarrow V(H)$ between the vertex sets, which preserves the adjacency relation, $(f(u), f(v)) \in E(H)$ for all $(u, v) \in E(G)$ (for which we also write $u \sim v$ ). We denote the category of graphs and graph homomorphisms by $\mathcal{G}$.

The graph 1 consisting of one vertex and a loop is a final object in the category of graphs. Any two graphs $G$ and $H$ have a categorical product $G \times H$. For every graph $G$, the functor $\bullet \times G$ has a right 
adjoint $[G, \bullet]$, i.e. there is a natural equivalence $\mathcal{G}(Z \times G, H) \cong \mathcal{G}(Z,[G, H])$. The graph $[G, H]$ is also written $H^{G}$ and called an exponential graph. The graph homomorphisms from $G$ to $H$ correspond to the looped vertices of $[G, H]$ in accordance with $\mathcal{G}(G, H) \cong \mathcal{G}(\mathbf{1} \times G, H) \cong \mathcal{G}(\mathbf{1},[G, H])$.

For graphs $G$ and $H$ we define a poset

$$
\operatorname{Hom}(G, H)=\left\{f \in(\mathcal{P}(V(H)) \backslash\{\varnothing\})^{V(G)}: f(u) \times f(v) \subset E(H) \text { f. a. }(u, v) \in E(G)\right\}
$$

with $f \leq g$ if and only if $f(u) \subset g(u)$ for all $u \in V(G)$. Hom is a functor from $\mathcal{G}^{\text {opp }} \times \mathcal{G}$ to the category of posets and order preserving maps. $\operatorname{Hom}(G, H)$ is the face poset of a cell complex first described in Babson and Kozlov (2006). The special case $\operatorname{Hom}(\mathbf{1}, H)$ is the poset of cliques of looped vertices of $H$. The atoms of $\operatorname{Hom}(G, H)$ correspond to the graph homomorphisms from $G$ to $H$. More is true: There is a natural homotopy equivalence $|\operatorname{Hom}(G, H)| \simeq|\operatorname{Hom}(\mathbf{1},[G, H])|$ induced by a poset map which preserves atoms and with a homotopy inverse of the same kind. Also $|\operatorname{Hom}(\mathbf{1}, \bullet)|$ preserves products up to such an equivalence, see Dochtermann (2009b). More formal properties of Hom can be derived from the above facts, in particular the existence of a map

$$
\operatorname{Hom}(G, H) \times \operatorname{Hom}(H, Z) \rightarrow \operatorname{Hom}(G, Z)
$$

which on atoms corresponds to composition of graph homomorphisms and has all the expected properties. Of course such a map is also easy to write down explicitly, it was first used in Schultz (2009).

Another construction that we will use takes a poset $P$ and assigns to it a reflexive graph $P^{1}$. The vertices of $P^{1}$ are the atoms of $P$, and two atoms are adjacent in $P^{1}$, if and only if they have a common upper bound in $P$. This construction played in important role in Dochtermann and Schultz (2010) and we will use several results from there. Usually $P$ is the face poset of a cell complex $X$, in which case we also write $X^{1}$ instead of $P^{1}$ and call it the looped 1-skeleton of $X$.

\section{The Bárány-Schrijver construction Stable Kneser graphs and the alternating oriented matroid}

Our starting point is the realization that parts of the construction which was used by Bárány (1978) to prove $\chi\left(K G_{n, k}\right)=k+2$ without using graph complexes and later refined by Schrijver (1978) to prove $\chi\left(S G_{n, k}\right)=k+2$ can also be used to tell us something about graph complexes, namely the existence of an equivariant map $\mathbb{S}^{k} \rightarrow C_{2} \operatorname{Hom}\left(K_{2}, S G_{n, k}\right)$. This was also implicitly used by Ziegler (2002) in a combinatorial proof of $\chi\left(S G_{n, k}\right)=k+2$, which built open a combinatorial proof of Kneser's conjecture by Matoušek (2004).

Schrijver uses vectors $v_{0}, \ldots, v_{2 n+k-1}$ on the moment curve to define a covering of $\mathbb{S}^{k}$ by the system of sets $\left(\left\{x \in \mathbb{S}^{k}:(-1)^{j}\left\langle x, v_{j}\right\rangle>0 \text { f.a. } j \in S\right\}\right)_{S \in V\left(S G_{n, k}\right)}$ and use the Borsuk-Ulam theorem to conclude that $\chi\left(S G_{n, k}\right) \geq k+2$.

We define the alternating oriented matroid $C^{m, k+1}, m>k \geq 0$, to be the oriented matroid associated to the vector configuration $v_{0}, \ldots, v_{m-1} \in \mathbb{R}^{k+1}$ with $v_{j}=\left(1, t_{j}, \ldots, t_{j}^{k}\right)$ for some real numbers $t_{0}<$ $t_{1}<\cdots<t_{m-1}$ (Björner et al., 1999, 9.4). The set of non-zero covectors is

$$
\mathcal{L}\left(C^{m, k+1}\right)=\left\{\left(\operatorname{sign}\left\langle x, v_{0}\right\rangle, \ldots, \operatorname{sign}\left\langle x, v_{m-1}\right\rangle\right): x \in \mathbb{R}^{k+1} \backslash\{0\}\right\} \subset\{-1,0,+1\}^{m} .
$$

We regard $\mathcal{L}\left(C^{m, k+1}\right)$ as a poset with the partial order induced by the partial order on $\{-1,0,+1\}$ given by $s \leq s^{\prime} \Longleftrightarrow s=0 \vee s=s^{\prime}$. The elements of $\mathcal{L}\left(C^{m, k+1}\right)$ are exactly the sign vectors with at most 
$k$ sign changes. By this we mean those sign vectors obtained as $(\operatorname{sign} p(0), \ldots, \operatorname{sign} p(m-1))$ with $p$ a polynomial of degree $k$. The minimal elements, called cocircuits, are those with exactly $k$ zeros (and hence a sign change at every zero, to be interpreted as above).

2.1 Proposition and Definition. Let $n \geq 1, k \geq 0$ and $m=2 n+k$. For $s=\left(s_{0}, \ldots, s_{m-1}\right) \in$ $\mathcal{L}\left(C^{m, k+1}\right)$ let the sets $S_{0}(s), S_{1}(s) \subset\{0, \ldots, m-1\}$ be defined by

$$
S_{l}(s)=\left\{j:(-1)^{j} s_{j}=(-1)^{l}\right\} .
$$

Then

$$
\begin{aligned}
\mathcal{L}\left(C^{m, k+1}\right) & \rightarrow \operatorname{Hom}\left(K_{2}, S G_{n, k}\right) \\
s & \mapsto\left(l \mapsto\left\{T \in V\left(S G_{n, k}\right): T \subset S_{l}(s)\right\}\right),
\end{aligned}
$$

with $V\left(K_{2}\right)=\{0,1\}$, is a well-defined order preserving map.

Proof: Denote the map by $g$. For $s \in \mathcal{L}\left(C^{m, k+1}\right)$ obviously $S_{0}(s) \cap S_{1}(s)=\emptyset$, so $\left(T_{0}, T_{1}\right) \in E\left(S G_{n, k}\right)$ for all $T_{l} \in g(s)_{l}$. We only have to check that $g(s)_{l} \neq \varnothing$, and since $g$ is order preserving by construction, we can assume that $s$ is a cocircuit. But then $s$ contains exactly $k$ zeros. Let $i_{0}<i_{1}<\cdots<i_{2 n-1}$ be the indices at which $s$ is non-zero. That $s$ has sign changes at exactly the zeros means that $s_{i_{j+1}}=$ $(-1)^{i_{j+1}-i_{j}-1} s_{i_{j}}$, i.e. $(-1)^{i_{j+1}} s_{i_{j+1}}=-(-1)^{i_{j}} s_{i_{j}}$. Therefore $S_{0}(s)$ and $S_{1}(s)$ are interleaved $n$-sets and $S_{0}(s), S_{1}(s) \in V\left(S G_{n, k}\right)$.

2.2 Proposition and Definition. Let $n \geq 1, k \geq 0$ and $m=2 n+k$ and $\left(v_{j}\right)$ the vector configuration above or any other vector configuration realizing $C^{m, k+1}$.

The covector poset $\mathcal{L}\left(C^{m, k+1}\right)$ is the face poset of a cell decomposition of $\mathbb{S}^{k}$, where the open cell corresponding to $s \in \mathcal{L}\left(C^{m, k+1}\right)$ is $\left\{x \in \mathbb{S}^{k}: \operatorname{sign}\left\langle x, v_{j}\right\rangle=s_{j}\right\}$. Therefore the poset map of Definition 2.1 induces a continuous map

$$
f: \mathbb{S}^{k} \rightarrow\left|\operatorname{Hom}\left(K_{2}, S G_{n, k}\right)\right| .
$$

If we write the group with 2 elements as $C_{2}=\{e, \tau\}$ and have $C_{2}$ operate via the antipodal map on $\mathbb{S}^{k}$ and via the isomorphism $\operatorname{Aut}\left(K_{2}\right) \cong C_{2}$ on $\operatorname{Hom}\left(K_{2}, S G_{n, k}\right)$, then the map $f$ satisfies $f(\tau \cdot x)=\tau \cdot f(x)$ for all $x \in \mathbb{S}^{k}$.

\section{The action of the dihedral group}

The map of Definition 2.2 turns out to be useful in studying $\operatorname{Hom}\left(K_{2}, S G_{n, k}\right)$. Since we are interested in the action of the automorphism group of $S G_{n, k}$ on $\operatorname{Hom}\left(K_{2}, S G_{n, k}\right)$, we will choose different vectors in the construction to obtain an equivariant map with respect to an orthogonal action of $\operatorname{Aut}\left(S G_{n, k}\right)$ on $\mathbb{S}^{k}$. We do this by replacing the moment curve with the trigonometric moment curve.

2.3 Definition. For $m \geq 2$ let $D_{2 m}=\left\langle\sigma, \rho \mid \rho^{2}=\sigma^{m}=(\sigma \rho)^{2}=1\right\rangle$ denote the dihedral group with $2 m$ elements.

For $m=2 n+k$ we define a right $D_{2 m}$ action on $K G_{n, k}$ by

$$
S \cdot \sigma=\{j+1: j \in S\}, \quad S \cdot \rho=\{-j: j \in S\},
$$

where all arithmetic is modulo $m$. The subgraph $S G_{n, k}$ is invariant under this action. We also set $C_{2}=$ $\left\langle\tau \mid \tau^{2}=1\right\rangle$ and have this group act nontrivially on $K_{2}$ from the right. Since Hom is contravariant in 
the first and covariant in the second parameter, this defines a left $C_{2}$-action and a right $D_{2 m}$-action on $\operatorname{Hom}\left(K_{2}, S G_{n, k}\right)$, and these commute.

For $n>1$ the homomorphism $D_{2 m} \rightarrow \operatorname{Aut}\left(S G_{n, k}\right)$ given by this action has been shown to be an isomorphism by Braun (2010).

Our goal is to choose vectors $v_{i}$ in Definition 2.2 in such a way that that map becomes $D_{2 m}$-equivariant with respect to an easy to define $D_{2 m}$-action on $\mathbb{S}^{k}$. We achieve this by essentially replacing the moment curve by the trigonometric moment curve.

2.4 Definition. Let $n \geq 1, k \geq 0, m=2 n+k$, We define orthogonal actions on $\mathbb{R}^{k+1}$ and vectors in $\mathbb{R}^{k+1}$. In the following $R_{\phi}=\left(\begin{array}{cc}\cos \phi & -\sin \phi \\ \sin \phi & \cos \phi\end{array}\right)$. First we set $\tau \cdot x=-x$.

For $k=2 r$ we set

$$
\begin{aligned}
x \cdot \sigma & =-\operatorname{diag}\left(1, R_{2 \pi / m}, R_{4 \pi / m}, \ldots, R_{k \pi / m}\right) \cdot x, \quad x \cdot \rho=\operatorname{diag}(1,1,-1, \ldots, 1,-1) \cdot x, \\
v_{j} & =(-1)^{j}(1,1,0, \ldots, 1,0) \cdot \sigma^{j} .
\end{aligned}
$$

For $k=2 r+1$ we set

$$
\begin{aligned}
x \cdot \sigma & =-\operatorname{diag}\left(R_{\pi / m}, R_{3 \pi / m}, \ldots, R_{k \pi / m}\right) \cdot x, \quad x \cdot \rho=\operatorname{diag}(1,-1, \ldots, 1,-1) \cdot x, \\
v_{j} & =(-1)^{j}(1,0, \ldots, 1,0) \cdot \sigma^{j} .
\end{aligned}
$$

We denote $\mathbb{R}^{k+1}$ equipped with the orthogonal right action of $D_{2 m}$ defined above by $W_{n, k}$. The unit sphere in $W_{n, k}$ is denoted by $\mathbb{S}\left(W_{n, k}\right)$.

It is now not too hard to check that the system $\left(v_{j}\right)_{0 \leq j<m}$ realizes $C^{m, k+1}$ and that using it in Definition 2.1 makes the resulting map $D_{2 m}$-equivariant.

2.5 Theorem. Let $n \geq 1, k \geq 0$, and $m=2 n+k$. There is a continuous map $f: \mathbb{S}\left(W_{n, k}\right) \rightarrow$ $\left|\operatorname{Hom}\left(K_{2}, S G_{n, k}\right)\right|$ which is equivariant with respect to the actions of $C_{2}$ and $D_{2 m}$ defined above.

\section{Homotopy test graphs and $\Gamma$-test graphs}

In addition to Definition 1.5 of a homotopy test graph we also define a $\Gamma$-test graph for a group $\Gamma$. We denote by $E_{r} \Gamma$ any $r$-dimensional, $(r-1)$-connected $\mathrm{CW}$-space with a free $\Gamma$-action, and by $E \Gamma$ any contractible CW-space with a free $\Gamma$-action. We write $B_{r} \Gamma=E_{r} \Gamma / \Gamma$ and $B \Gamma=E \Gamma / \Gamma$ for the coresponding orbit spaces.

3.1 Definition. Let $T$ be a graph with a right action of a finite group $\Gamma$. We call $T$ a $\Gamma$-test graph, if for all loopless graphs $G$ and integers $r \geq 0$ such that there exists an equivariant map $E_{r} \Gamma \rightarrow_{\Gamma}|\operatorname{Hom}(T, G)|$ the inequality $\chi(G) \geq \chi(T)+r$ holds.

3.2 Proposition. Let $T$ be a graph with a right action of a finite group $\Gamma$. If $T$ is a $\Gamma$-test graph, then $T$ is a homotopy test graph.

Proof: If $|\operatorname{Hom}(T, G)|$ is $(r-1)$-connected, then there is an equivariant map $E_{r} \Gamma \rightarrow_{\Gamma}|\operatorname{Hom}(T, G)|$, since $E_{r} \Gamma$ is an $r$-dimensional free $\Gamma$-space.

We have announced a partial converse of this result in Theorem 1.10 It follows from the following theorem by setting $\Gamma=\operatorname{Aut}(T), s=\chi(T)+r$ and considering the equivalence of (ii) and (iii). 
In (i), $X^{1}$ denotes the looped 1-skeleton of $X$ and $T \times_{\Gamma} X^{1}$ the orbit graph of the diagonal action of $\Gamma$ on $T \times X^{1}$. This construction is explored in Dochtermann and Schultz (2010).

3.3 Theorem. Let $T$ be a finite, connected graph equipped with a right action of a finite group $\Gamma$. Let $r \geq 0, s \geq 1$. Then each of the following statements implies the next.

(i) For every $\Gamma$-invariant triangulation $X$ of $E_{r} \Gamma$ the inequality $\chi\left(T \times_{\Gamma} X^{1}\right) \geq s$ holds.

(ii) For all graphs $G$ such that there is a $\Gamma$-equivariant map $E_{r} \Gamma \rightarrow|\operatorname{Hom}(T, G)|$ the inequality $\chi(G) \geq s$ holds.

(iii) For all graphs $G$ such that $|\operatorname{Hom}(T, G)|$ is $(r-1)$-connected the inequality $\chi(G) \geq s$ holds.

If $T$ is vertex critical and $\Gamma=\operatorname{Aut}(T)$, then (iii) implies (i) and all of the statements are equivalent.

Proof: The implication (i) $\Longrightarrow$ (ii) is a standard application of the techniques developped in Dochtermann and Schultz (2010) and earlier papers, the implication (ii) $\Longrightarrow$ (iii) is immediate as in Proposition 3.2

To prove the implication (iii) $\Longrightarrow$ (i) we assume that a triangulation $X$ of $E_{r} \Gamma$ is given. If we obtain $Y$ from $X$ by repeated barycentric subdivision, then $Y$ is also $\Gamma$-invariant, and there is an equivariant graph homomorphism $Y^{1} \rightarrow_{\Gamma} X^{1}$. If the subdivision $Y$ is fine enough, then

$$
\left|\operatorname{Hom}\left(T, T \times_{\Gamma} Y^{1}\right)\right| \simeq|\operatorname{Hom}(T, T)| \times_{\Gamma}\left|\operatorname{Hom}\left(T, Y^{1}\right)\right|
$$

by Sec. 5.2 of Dochtermann and Schultz (2010). Since we assumed $T$ to be vertex critical, the only endomorphisms of $T$ are the automorphisms. It also follows that $\operatorname{Hom}(T, T)$ is 0-dimensional, $\operatorname{Hom}(T, T) \cong \operatorname{Aut}(T)$. We assumed $\Gamma=\operatorname{Aut}(T)$. Therefore

$$
|\operatorname{Hom}(T, T)| \times_{\Gamma}\left|\operatorname{Hom}\left(T, Y^{1}\right)\right| \approx \operatorname{Aut}(T) \times_{\operatorname{Aut}(T)}\left|\operatorname{Hom}\left(T, Y^{1}\right)\right| \approx\left|\operatorname{Hom}\left(T, Y^{1}\right)\right| .
$$

But by Thm 3.1 of Dochtermann (2009a), since $T$ is connected, and again if $Y$ is a fine enough subdivision,

$$
\left|\operatorname{Hom}\left(T, Y^{1}\right)\right| \simeq|Y| \approx E_{r} \Gamma .
$$

Therefore $\left|\operatorname{Hom}\left(T, T \times_{\Gamma} Y^{1}\right)\right|$ is $(r-1)$-connected. Hence $\chi\left(T \times_{\Gamma} X^{1}\right) \geq \chi\left(T \times_{\Gamma} Y^{1}\right) \geq s$.

\section{Stable Kneser graphs which are test graphs}

The approach that we use to show that for certain $n, k$ the graph $S G_{n, k}$ is a test graph is similar to that used for odd cycles $(k=1)$ in Schultz (2009).

We assume the existence of an equivariant map $E_{r} D_{2 m} \rightarrow_{D_{2 m}} \operatorname{Hom}\left(S G_{n, k}, G\right)$. In Theorem 2.5 we have constructed a map $\mathbb{S}\left(W_{n, k}\right) \rightarrow_{D_{2 m}} \operatorname{Hom}\left(K_{2}, S G_{n, k}\right)$. It is known that $\operatorname{Hom}\left(K_{2}, K_{k+r+1}\right) \simeq_{C_{2}}$ $\mathbb{S}^{k+r-1}$. So if $\chi(G)<k+r+2$, then we obtain a map

$$
\begin{aligned}
\mathbb{S}\left(W_{n, k}\right) \times_{D_{2 m}} E_{r} D_{2 m} \rightarrow_{C_{2}} \operatorname{Hom}\left(K_{2},\right. & \left.S G_{n, k}\right) \times_{D_{2 m}} \operatorname{Hom}\left(S G_{n, k}, G\right) \\
& \rightarrow C_{2} \operatorname{Hom}\left(K_{2}, G\right) \rightarrow_{C_{2}} \operatorname{Hom}\left(K_{2}, K_{k+r+1}\right) \simeq_{C_{2}} \mathbb{S}^{k+r-1},
\end{aligned}
$$

the second arrow being the map (2) Now $\mathbb{S}\left(W_{n, k}\right) \times_{D_{2 m}} E_{r} D_{2 m}$ is the total space of the sphere bundle associated to the euclidean vector bundle $W_{n, k} \times{ }_{D_{2 m}} E_{r} D_{2 m} \rightarrow B_{r} D_{2 m}$, and vector bundle theory yields obstructions to the existence of an equivariant map as above. 
4.1 Theorem. Let $\Gamma$ be a finite group and $\mathbb{R}^{k+1}$ be equipped with an orthogonal right $\Gamma$-action. Let $\xi$ be the $(k+1)$-dimensional bundle $\mathbb{R}^{k+1} \times_{\Gamma} E \Gamma \rightarrow B \Gamma$. Consider $\mathbb{S}^{k} \times_{\Gamma} E_{r}$ as a (free) $C_{2}$-space via the antipodal action on $\mathbb{S}^{k}$. If there is an equivariant map

$$
\mathbb{S}^{k} \times_{\Gamma} E_{r} \Gamma \rightarrow_{C_{2}} \mathbb{S}^{r+k-1}
$$

for some $r \geq 0$, then it follows that $\bar{w}_{r}(\xi)=0 \in H^{r}\left(B \Gamma ; \mathbb{Z}_{2}\right)=H^{r}\left(\Gamma ; \mathbb{Z}_{2}\right)$.

Here $\bar{w}_{r}(\xi)$ denotes the $r$-th dual Stiefel-Whitney class of the bundle $\xi$. These classes and the StiefelWhitney classes are related by $1=w(\xi) \bar{w}(\xi)=\sum_{r \geq 0} w_{r}(\xi) \cdot \sum_{r \geq 0} \bar{w}_{r}(\xi)$. We see that it is important to calculate the Stiefel-Whitney classes of the following bundles.

4.2 Definition. Let $n \geq 1, k \geq 0, m=2 n+k$. We denote the $(k+1)$-dimensional vector bundle $W_{n . k} \times_{D_{2 m}} E D_{2 m} \rightarrow B D_{2 m}$ by $\xi_{n, k}$.

Theorem 4.1 and the discussion preceding it already prove Proposition 1.8 .

With some knowledge of the $\mathbb{Z}_{2}$-cohomology of the dihedral groups, the classes $w_{r}\left(\xi_{n, k}\right)$ can more or less directly be read off from the action described in Definition 2.4. The results are the following.

4.3 Proposition. Let $k=2 r+1, r \geq 0, n>0$, and $m=2 n+k$. Then

$$
w\left(\xi_{n, k}\right)=(1+\alpha)^{r+1} \in H^{*}\left(D_{2 m} ; \mathbb{Z}_{2}\right) \cong \mathbb{Z}_{2}[\alpha], \quad|\alpha|=1 .
$$

Here $\mathbb{Z}_{2}[\alpha]$ refers to the free graded $\mathbb{Z}_{2}$-algebra with one generator $\alpha$, which is of degree $1(|\alpha|=1)$.

Let $k=2 r, r \geq 1, n>0, n \equiv r+1(\bmod 2)$, and $m=2 n+k$. Then

$$
\begin{aligned}
& w\left(\xi_{n, k}\right)=(1+\alpha)(1+\beta)^{\lceil r / 2\rceil}((1+\alpha)(1+\alpha+\beta))^{\lfloor r / 2\rfloor} \\
& \in H^{*}\left(D_{2 m} ; \mathbb{Z}_{2}\right) \cong \mathbb{Z}_{2}[\alpha, \beta], \quad|\alpha|=|\beta|=1 .
\end{aligned}
$$

Let $k=2 r, r \geq 1, n>0, n \equiv r(\bmod 2), m=2 n+k$. Then

$$
\begin{aligned}
w\left(\xi_{n, k}\right)=(1+y)(1+x+y+u)^{\lceil r / 2\rceil}(1+x+y)^{\lfloor r / 2\rfloor} & \\
& \in H^{*}\left(D_{2 m} ; \mathbb{Z}_{2}\right) \cong \mathbb{Z}_{2}[x, y, u] /(x y), \quad|x|=|y|=1,|u|=2 .
\end{aligned}
$$

From these and Proposition 1.8 we obtain Theorem 1.9 by calculation. We show the most interesting example.

4.4 Corollary. Let $s \geq 1$, then $S G_{2 s, 4}$ is a $D_{2 m}$-test graph.

Proof: We want to show that $\bar{w}_{r}\left(\xi_{n, k}\right) \neq 0$ for all $r$. If $j$ is the homomorphism from the ring $H^{*}\left(D_{2 m} ; \mathbb{Z}_{2}\right)$ to its quotient by the ideal $(x-y)$, then

$$
\begin{aligned}
& j\left(w\left(\xi_{2 s, 4}\right)\right)=(1+x)(1+2 x+u)(1+2 x)=(1+x)(1+u), \\
& j\left(\bar{w}\left(\xi_{2 s, 4}\right)\right)=(1+x)^{-1}(1+u)^{-1}=(1+x) \sum_{i \geq 0} u^{i},
\end{aligned}
$$

i.e. $\left.j\left(\bar{w}_{2 \ell}\right)\left(\xi_{2 s, 4}\right)\right)=u^{\ell} \neq 0, j\left(\bar{w}_{2 \ell+1}\left(\xi_{2 s, 4}\right)\right)=x u^{\ell} \neq 0$.

4.5 Remark. Since the homomorphism $j$ is actually induced by the inclusion of a cyclic subgroup $\Gamma \subset$ $D_{2 m}$, the graph $S G_{2 s, 4}$ is a $\Gamma$-test graph for that $\Gamma$. 


\section{Stable Kneser graphs and Borsuk graphs}

We investigate the relationship between Borsuk graphs (Definition 1.6) of spheres and stable Kneser graphs. In Section 2 we have constructed a map $\mathbb{S}\left(W_{n, k}\right) \rightarrow_{C_{2} \times D_{2 m}}\left|\operatorname{Hom}\left(K_{2}, S G_{n, k}\right)\right|$ via a poset map

$$
\mathcal{L}\left(C^{m, k+1}\right) \rightarrow_{C_{2} \times D_{2 m}} \operatorname{Hom}\left(K_{2}, S G_{n, k}\right)
$$

which sends atoms to atoms and therefore gives rise to a graph homomorphism

$$
\mathcal{L}\left(C^{m, k+1}\right)^{1} \rightarrow_{C_{2} \times D_{2 m}} \operatorname{Hom}\left(K_{2}, S G_{n, k}\right)^{1} \cong C_{2} \times D_{2 m}\left[K_{2}, S G_{n, k}\right]
$$

which in turn is adjoint to a graph homomorphism

$$
K_{2} \times_{C_{2}} \mathcal{L}\left(C^{m, k+1}\right)^{1} \rightarrow{ }_{D_{2 m}} S G_{n, k} .
$$

Regarding $\mathcal{L}\left(C^{m, k+1}\right)$ as the face poset of a cellular decomposition of $\mathbb{S}^{k}$, the graph $K_{2} \times_{C_{2}} \mathcal{L}\left(C^{m, k+1}\right)^{1}$ can be described as the graph whose vertices are the vertices of that cell complex and in which the neighbours of a vertex are those vertices which share a face with its antipodal vertex. This could be called the Borsuk graph of this $C_{2}$-cell complex and is a discrete analog of the $\varepsilon$-Borsuk graph defined in Definition 1.6 We will now construct a graph homomorphism in the other direction and using $B_{\varepsilon}\left(\mathbb{S}\left(W_{n, k}\right)\right)$.

5.1 Lemma. Let $m=2 n+k, S \in V\left(S G_{n, k}\right)$ and $\left(v_{i}\right)_{0 \leq i<m}$ a system of vectors in $\mathbb{R}^{k+1}$ realizing the alternating oriented matroid $C^{m, k+1}$. Then $\sum_{i \in S}(-1)^{i} v_{i} \neq 0$.

Proof: Assume that $S \subset\{0, \ldots, m-1\}$ and $\sum_{i \in S}(-1)^{i} v_{i}=0$. Since every minimal linear dependency of the vectors $v_{i}$ is of cardinality $k+2$ and has coefficients with alternating signs (see the proof of Prop. 9.4.1 in Björner et al. (1999)) there are $j_{0}<j_{1}<\cdots<j_{k+1}$ with $\left\{j_{s}\right\} \subset S$ and $(-1)^{j_{s+1}}=$ $(-1)^{j_{s}+1}$ for $0 \leq s \leq k$. If $S$ is a stable set, i.e. one which does not contain consecutive elements, then this implies $m \geq 2|S|+k+1$ and hence $|S|<n$.

This justifies the following definition.

5.2 Definition. For $S \in V\left(S G_{n, k}\right)$ let

$$
v(S):=\frac{\sum_{i \in S}(-1)^{i} v_{i}}{\left\|\sum_{i \in S}(-1)^{i} v_{i}\right\|},
$$

where $v_{i} \in \mathbb{R}^{k+1}$ is as in the proof of Theorem 2.5

5.3 Proposition. Let $k \geq 0$ and $\varepsilon>0$. Then there is an $N \in \mathbb{N}$ such that for all $n \geq N$ the function $v: V\left(S G_{n, k}\right) \rightarrow \mathbb{S}^{k}$ is a $D_{2 m}$-equivariant graph homomorphism

$$
S G_{n, k} \rightarrow_{D_{2 m}} B_{\varepsilon}\left(\mathbb{S}\left(W_{n, k}\right)\right) .
$$

Proof (sketch): Equivariance follows from the choice of the vectors $v_{i}$. For the homomorphism property, we have to show that there is an $N$ such that $\|v(S)+v(T)\|<\varepsilon$ for all $(S, T) \in E\left(S G_{n, k}\right)$ with $n \geq N$. Now for fixed $k$ and large $n$ the sets $S$ and $T \sigma$ must have a small symmetric difference, if $S$ and $T$ are disjoint and both stable. Now, again for large $n,(-1)^{i} v_{i}$ is close to $-(-1)^{i+1} v_{i+1}$. This shows that $\sum_{i \in S}(-1)^{i} v_{i}+\sum_{i \in T}(-1)^{i} v_{i}$ will indeed be small. It remains to show that neither of the two summands can have small norm. This requires a quantitative version of Lemma 5.1 for this particular choice of $\left(v_{i}\right)$, which can be obtained using some analysis. 


\section{Stable Kneser graphs which are not test graphs}

We want to prove Proposition 1.11, which states that the vanishing of a topological obstruction lets us indeed prove that certain stable Kneser graphs are not test graphs. We first state a purely topological result and then turn the existence of the vector bundle guaranteed by it into the existence of a graph with lower chromatic number than expected.

6.1 Proposition. Let $\xi$ be a vector bundle over an $r$-dimensional finite simplicial complex $X$ and $\bar{w}_{r}(\xi)=$ 0 . If $r=1$ or $r \equiv 0(\bmod 2)$, then there is an $(r-1)$-dimensional vector bundle $\eta$ over $X$ such that $\xi \oplus \eta$ is trivial.

6.2 Proposition. Let $k \geq 0, r>0$. Then there is an $\varepsilon>0$ such that the following holds.

Let $\Gamma$ be a finite group which acts from the right on $\mathbb{R}^{k+1}$ by orthogonal maps. Let $X$ be a finite simplicial complex with a free $\Gamma$-action and let $\xi$ be the vector bundle $\mathbb{R}^{k+1} \times_{\Gamma}|X| \rightarrow|X| / \Gamma$. If there exists an $(r-1)$-dimensional vector bundle $\eta$ over $|X| / \Gamma$ such that $\xi \oplus \eta$ is trivial, then there is a $\Gamma$-invariant subdivision $Y$ of $X$ such that

$$
\chi\left(B_{\varepsilon}\left(\mathbb{S}^{k}\right) \times_{\Gamma} Y^{1}\right)<k+2+r .
$$

Proof: We choose a covering $\left(A_{i}\right)_{i=0, \ldots, k+r}$ of $\mathbb{S}^{k+r-1}$ by closed subsets such that no $A_{i}$ contains a pair of antipodal points. Let $D:=\min _{i} \operatorname{dist}\left(A_{i},-A_{i}\right)>0,0<\varepsilon<D$ and $\varepsilon^{\prime}:=D-\varepsilon$.

Since $\Gamma$ acts by orthogonal maps, the bundle $\xi$ is a Euclidean vector bundle. The bundle $\eta$ can be made into a Euclidean vector bundle, and the $r+l$ linear independent sections of $\xi \oplus \eta$ which define the trivialization can be made orthogonal using Gram-Schmidt. Therefore there is a continuous map $E(\xi)=\mathbb{R}^{k+1} \times_{\Gamma}|X| \rightarrow \mathbb{R}^{k+r}$ such that the restriction to each fibre of $\xi$ is a linear isometry. Denoting the space of linear isometries from $\mathbb{R}^{k+1}$ to $\mathbb{R}^{k+r}$ by Iso $\left(\mathbb{R}^{k+1}, \mathbb{R}^{k+r}\right)$ and viewing it as a $\Gamma$-space via the action on $\mathbb{R}^{k+1}$, this is equivalent to the existence of an equivariant continuous map

$$
f:|X| \rightarrow_{\Gamma} \operatorname{Iso}\left(\mathbb{R}^{k+1}, \mathbb{R}^{k+r}\right) .
$$

We let $Y$ be a subdivision of $X$ such that for all pairs $y, y^{\prime}$ of neighbouring vertices of $Y$ we have $\| f(y)-$ $f\left(y^{\prime}\right) \|<\varepsilon^{\prime}$, where $\|\bullet\|$ denotes the operator norm. We define

$$
\begin{aligned}
c: \mathbb{S}^{k} \times V(Y) & \rightarrow\{0, \ldots, k+r\}, \\
(v, y) & \mapsto \min \{i: f(y)(v)\} .
\end{aligned}
$$

Now if $v, v^{\prime} \in \mathbb{S}^{k},\left\|v+v^{\prime}\right\|<\varepsilon$, and $y, y^{\prime} \in V(Y),\left\{y, y^{\prime}\right\} \in Y$, then

$$
\operatorname{dist}\left(A_{c(v, y)},-A_{c\left(v^{\prime}, y^{\prime}\right)}\right) \leq\left\|f(y)(v)-\left(-f\left(y^{\prime}\right)\left(v^{\prime}\right)\right)\right\| \leq\left\|f(y)-f\left(y^{\prime}\right)\right\|+\left\|v-\left(-v^{\prime}\right)\right\|<\varepsilon^{\prime}+\varepsilon=D
$$

and hence $c(v, y) \neq c\left(v^{\prime}, y^{\prime}\right)$. This shows that the function $c$ is a graph homomorphism $B_{\varepsilon}\left(\mathbb{S}^{k}\right) \times Y^{1} \rightarrow$ $K_{k+r+1}$. Since for $\gamma \in \Gamma$ we have $f(\gamma y)(v)=(\gamma f)(v)=f(v \gamma)$, we have $c(v, \gamma y)=c(v \gamma, y)$, and $[(v, y)] \mapsto c(v, y)$ defines a $(k+1+r)$-colouring of $B_{\varepsilon}\left(\mathbb{S}^{k}\right) \times_{\Gamma} Y^{1}$.

Putting everything together yields the following more explicit version of Proposition 1.11

6.3 Proposition. Let $k, r \geq 1$ and $r=1$ or $r \equiv 0(\bmod 2)$. Then there is an $N \geq 2$ such that for all $n \geq N$ with $\bar{w}_{r}\left(\xi_{n, k}\right)=0$ there is a graph $G$ such that $\operatorname{Hom}\left(S G_{n, k}, G\right)$ is $(r-1)$-connected and $\chi(G)<k+2+r$. 
Proof: Given $k$ and $r$ we choose $\varepsilon>0$ as in Proposition 6.2 and $N$ as in Proposition 5.3 Now given $n \geq N, m=2 n+k$, there is an equivariant graph homomorphism $S G_{n, k} \rightarrow_{D_{2 m}} B_{\varepsilon}\left(W_{n, k}\right)$ by Proposition 5.3 Now if $\bar{w}_{r}\left(\xi_{n, k}\right)=0$ then by Proposition 6.1 and Proposition 6.2 there is a $D_{2 m^{-}}$invariant triangulation $Y$ of $E_{r} D_{2 m}$ such that $\chi\left(B_{\varepsilon}\left(W_{n, k}\right) \times_{D_{2 m}} Y^{1}\right)<k+2+r$ and therefore $\chi\left(S G_{n, k} \times_{D_{2 m}} Y^{1}\right)<k+r+2$. Since stable Kneser graphs are vertex critical with respect to the chromatic number by a theorem of Schrijver (1978) and $\operatorname{Aut}\left(S G_{n, k}\right)=D_{2 m}$ for $n>1$ by a theorem of Braun (2010), we can invoke Theorem 3.3 to conclude the proof.

Calculations using Proposition 4.3 now show that Proposition 6.3 is applicable in the cases needed for Theorem 1.12,

\section{References}

E. Babson and D. N. Kozlov. Complexes of graph homomorphisms. Israel J. Math., 152:285-312, 2006.

E. Babson and D. N. Kozlov. Proof of the Lovász conjecture. Ann. of Math. (2), 165(3):965-1007, 2007.

I. Bárány. A short proof of Kneser's conjecture. J. Combin. Theory Ser. A, 25(3):325-326, 1978.

A. Björner and M. de Longueville. Neighborhood complexes of stable Kneser graphs. Combinatorica, 23 (1):23-34, 2003. Paul Erdôs and his mathematics (Budapest, 1999).

A. Björner, M. Las Vergnas, B. Sturmfels, N. White, and G. M. Ziegler. Oriented matroids, volume 46 of Encyclopedia of Mathematics and its Applications. Cambridge University Press, Cambridge, second edition, 1999.

B. Braun. Symmetries of the stable kneser graphs. Advances in Applied Mathematics, 45(1):12 - 14, 2010.

A. Dochtermann. The universality of Hom complexes. Combinatorica, 29(4):433-448, 2009a.

A. Dochtermann. Hom complexes and homotopy theory in the category of graphs. European J. Combin., 30(2):490-509, 2009b.

A. Dochtermann and C. Schultz. Topology of Hom complexes and test graphs for bounding chromatic number. To appear in Israel J. Math., arXiv:0907.5079v2 [math.CO], 2010.

L. Lovász. Kneser's conjecture, chromatic number, and homotopy. J. Combin. Theory Ser. A, 25(3): 319-324, 1978.

J. Matoušek. A combinatorical proof of Kneser's conjecture. Combinatorica, 24(1):163-170, 2004.

A. Schrijver. Vertex-critical subgraphs of Kneser graphs. Nieuw Arch. Wisk. (3), 26(3):454-461, 1978.

C. Schultz. Graph colorings, spaces of edges and spaces of circuits. Adv. Math., 221(6):1733-1756, 2009.

C. Schultz. The equivariant topology of stable Kneser graphs. To appear in J. Combin. Theory Ser. A, arXiv:1003.5688, 2010.

G. M. Ziegler. Generalized Kneser coloring theorems with combinatorial proofs. Invent. Math., 147(3): 671-691, 2002. 